\title{
纳米二氧化锰的制备及其应用研究进展
}

\author{
王金敏，于红玉，马董云 \\ (上海第二工业大学 工学部, 环境与材料工程学院, 上海 201209)
}

摘 要: 二氧化锰作为一种重要的过渡金属氧化物, 因其储量丰富、晶型多样、性能优异而备受关注。将二氧化锰 纳米化后, 其颗粒尺寸变小、比表面积变大、材料性能优化、应用领域得以拓宽。本文在引言部分从介绍二氧化锰 的应用着手, 指出纳米化和晶型多变对二氧化锰的结构和性能有着重要的影响。正文部分主要从纳米二氧化锰的制 备方法和纳米二氧化锰的应用两个方面对近年来的研究进展进行了总结和评述。(1)介绍了水热法、溶胶一凝胶法、 化学沉淀法、固相合成法等纳米二氧化锰的制备方法, 对各种制备方法的优点与缺点以及所制备纳米二氧化锰的形 貌与性能进行了总结。(2)综述了纳米二氧化锰在储能电极、电致变色器件、催化剂、生物传感器等领域的应用研 究进展。纳米二氧化锰可作为电池的正极材料和超级电容器的电极材料。通过调控二氧化锰的晶型和复合制备的 含锰复合氧化物用于锂离子电池的正极材料, 可提高电池的容量并改善循环稳定性。作为锂离子动力电池的正极材 料已有产业化应用, 在新能源汽车领域具有良好的应用前景。由于纯二氧化锰本身的颜色主要是在棕色和黄色之间 变化, 光调制幅度较小, 因此作为电致变色器件的电极材料, 通常将其与其它光调制幅度较大的材料进行复合使 用。如聚苯胺/二氧化锰杂化电致变色薄膜较纯聚苯胺薄膜在形貌、结构和电致变色性能上有巨大差异, 显示出更 高的光调制幅度、着色效率和循环稳定性。纳米二氧化锰在乙苯的催化转化和空气污染物的催化消除方面发挥重 要作用。纳米二氧化锰能够增大电流响应、降低检出限, 使检测的灵敏度大大提高, 近年来在生物传感器领域逐渐 被大家重视并得到广泛应用, 如二氧化锰纳米片辅助荧光偏振生物传感器可有效检测环境水样中 $\mathrm{Ag}^{+}, \mathrm{PtAu}^{-\mathrm{MnO}_{2}}$ 二元纳米结构修饰的石墨烯纸在非酶葡萄糖检测中表现出良好的传感性能。在结语部分, 分析了当前纳米二氧化锰 的制备和应用方面存在的问题, 指出了纳米二氧化锰在锂离子电池正极材料和电致变色器件中应用的发展方向, 并对其未来的发展前景进行了展望。

关 键 词: 纳米二氧化锰; 水热法; 储能电极; 电致变色

中图分类号: TQ174 文献标识码: A

\section{Progress in the Preparation and Application of Nanostructured Manganese Dioxide}

\author{
WANG Jinmin, YU Hongyu, MA Dongyun
}

(School of Environmental and Materials Engineering, College of Engineering, Shanghai Polytechnic University, Shanghai 201209, China)

Abstract: As an important transition metal oxide, manganese dioxide $\left(\mathrm{MnO}_{2}\right)$ has attracted more and more attention due to its abundant reserves, varied crystal types and excellent material properties. Nanostructured $\mathrm{MnO}_{2}$ has

收稿日期: 2020-03-02; 收到修改稿日期：2020-05-07

基金项目：国家自然科学基金(61775131, 61376009); 上海高校特聘教授(东方学者)岗位计划(2013-70) National Natural Science Foundation of China (61775131, 61376009); The Program for Professor of Special Appointment (Eastern Scholar) at Shanghai Institutions of Higher Learning (2013-70)

作者简介: 王金敏(1975-)，男，教授. E-mail: wangjinmin@sspu.edu.cn WANG Jinmin (1975-), male, professor. E-mail: wangjinmin@sspu.edu.cn 
smaller size and larger specific surface area, that makes it can further optimize its material properties and expand its application fields. In the introduction, this article starts with the introduction of the application of manganese dioxide, and points out that nanostructuring and variability in crystal form have an important influence on the structure and properties of manganese dioxide. The main text summarizes and reviews the research progress in recent years from two aspects: the preparation methods and the applications of nanostructured $\mathrm{MnO}_{2}$. (1) This paper introduces the progress in the preparation methods of nanostructured $\mathrm{MnO}_{2}$ including hydrothermal, Sol-Gel, chemical precipitation, solid-phase synthesis. Then the advantages and disadvantages of preparation methods, the morphologies and properties of nanostructured $\mathrm{MnO}_{2}$ are summarized. (2) The applications of nanostructured $\mathrm{MnO}_{2}$ including energy-storage electrodes, electrochromic devices, catalysts and bio-sensors are reviewed. Nanostructured $\mathrm{MnO}_{2}$ can be used as the cathode material of batteries and the electrode material of supercapacitors. Manganesecontaining composite oxides prepared by adjusting the crystal form of $\mathrm{MnO}_{2}$ and compounding are used as the cathode material of the lithium ion batteries, which can increase the capacities and improve the cycle stability of batteries. It has been industrialized as a cathode material for lithium-ion power batteries, and has good application prospects in the field of new energy vehicles. As the electrode material of electrochromic devices, $\mathrm{MnO}_{2}$ is usually used by combining with other materials with large optical modulations since the color of pure $\mathrm{MnO}_{2}$ mainly changes between brown and yellow and its optical modulation is small. For example, polyaniline $/ \mathrm{MnO}_{2}$ hybrid electrochromic film has a great difference in morphology, structure and electrochromic performance compared with pure polyaniline film, showing higher optical modulation, coloration efficiency and cycle stability. Nanostructured $\mathrm{MnO}_{2}$ plays important roles in the catalytic conversion of ethylbenzene and the catalytic elimination of air pollutants. Nanostructured $\mathrm{MnO}_{2}$ can increase the current response, reduce the detection limit, and greatly improve the sensitivity of detection. In recent years, it has been gradually paid attention to and widely used in the field of biosensors. For example, $\mathrm{MnO}_{2}$ nanosheets assisted fluorescence polarization biosensors can be effective in detection of $\mathrm{Ag}^{+}$in environmental water samples, $\mathrm{PtAu}-\mathrm{MnO}_{2}$ binary nanostructures modified graphene paper show good sensing performance in non-enzymatic glucose detection. In conclusion part, current existing problems are analyzed. The development direction of nanostructured $\mathrm{MnO}_{2}$ applied in lithium-ion battery cathode materials and electrochromic devices are pointed out. The future prospects for development of nanostructured $\mathrm{MnO}_{2}$ are discussed.

Key words: nanostructured manganese oxide; hydrothermal; energy-storage electrodes; electrochromism

\section{二氧化锰作为重要过渡金属氧化物具有储备丰} 富、环境友好、工作电压窗较宽等优点, 在电极材 料、电致变色、催化、生物传感器等领域都有广泛 的应用前景 ${ }^{[1-3]}$ 。二氧化锰经常含有少量的其它锰氧 化物和化合水 ${ }^{[4]}$, 分子式表示为 $\mathrm{MnO}_{x}(x<2)^{[5-6]}$ 。将 二氧化锰纳米化后, 其颗粒尺寸变小、比表面积增 $大^{[7]}$, 从而使离子的传输速率、催化效率等都有了进 一步的提升。除上述原因外, 纳米二氧化锰多变的 晶型也是它受到研究人员重视的主要原因之一。二 氧化锰有 $\alpha 、 \beta 、 \delta$ 等晶型，按 $\left[\mathrm{MnO}_{6}\right]$ 的连接方式不 同，又可分为链状或隧道状结构 ${ }^{[5-6]}$ 。这些原因促使 纳米二氧化锰在能源、光电、环境、生物医学等领 域有着广泛的应用。

纳米二氧化锰的制备方法多种多样, 主要包括 水热法 ${ }^{[8]}$ 、溶胶-凝胶法 ${ }^{[9]}$ 、化学沉淀法 ${ }^{[10]}$ 、固相合
成法等。由不同方法制备的纳米二氧化锰的晶相、 形貌、尺寸以及应用各有差异, 本文综述了纳米二 氧化锰的各种制备方法的优势与不足, 及其在储能 电池电极、电致变色器件、催化剂、生物传感器等 领域的应用研究进展。

\section{1 纳米二氧化锰的制备方法}

\section{1 水热法}

水热法是指在一定温度的密闭条件下, 以水为 溶剂的溶液产生压力，使得反应物之间发生反应从 而生成特定产物的制备方法 ${ }^{[11]}$ 。水热法有诸多优点, 例如不受模板的约束、初始反应物不必具有可溶性; 通过选择适合的温度、时间等, 可以较为方便地合 成纳米材料; 在密闭空间进行合成, 最大限度地减 
少了外界的干扰，极大地提高了生成产物的纯度， 是一种在实际操作中较为常用的合成方法。水热合 成法制备的纳米二氧化锰材料具有纯度高、分散性 好、晶型完整、晶粒大小可控等优点。但由于要保 持高温高压的合成环境, 较其它合成方法更繁琐。 Zhao 等 ${ }^{[12]}$ 将制备好的中空碳纳米纤维加入到 $\mathrm{KMnO}_{4}$ 溶液中, 经 $120{ }^{\circ} \mathrm{C}$ 水热反应 $2 \mathrm{~h}$ 合成了表面 有 $\delta-\mathrm{MnO}_{2}$ 纳米片的中空碳纳米纤维, 得到了可用 于高性能非对称超级电容器的超薄 $\mathrm{MnO}_{2}$ 纳米薄片/ 中空碳纳米纤维的纳米复合材料。 $\mathrm{Wu}$ 等 ${ }^{[13]}$ 利用沸 石咪唑酯框架材料作为自模板, 在碳布上水热制备 了 $\mathrm{MnO}_{2}$ 空心纳米片结构, 纳米片厚度为 $10 \mathrm{~nm}$ 左 右, 制备过程如图 1 所示。 $\mathrm{MnO}_{2}$ 空心多面体具有较 大的比表面积, 易于实现电解质的渗透、抑制体积 膨胀, 而碳布基板促进电子传输, 进一步提高了锌 离子的存储性能。 $\mathrm{Lu}$ 等 ${ }^{[14]}$ 以 $\mathrm{KMnO}_{4}$ 和乙醇混合溶 液为反应溶液, 经 $120{ }^{\circ} \mathrm{C}$ 水热反应 $2 \mathrm{~h}$ 后得到了直 径为 $12 \mathrm{~nm}$ 的 $\alpha-\mathrm{MnO}_{2}$ 纳米线，并将其应用在 $\mathrm{Li}-\mathrm{O}_{2}$ 电池的电催化中。利用水热合成方法可以制备形貌 各异的二氧化锰纳米结构, 除上述所提到的结构外, 还有纳米颗粒、纳米棒、纳米晶须、纳米管、纳米 花束以及球壳结构等 ${ }^{[15-20]}$ 。

\section{2 溶胶一凝胶法}

作为一种常见的材料合成工艺, 溶胶-凝胶法 主要是通过水解缩聚金属醇盐或无机盐并使其逐渐 变成凝胶，经过相应处理后得到粉体材料的合成方 法 ${ }^{[21]}$ 。溶胶一凝胶法制备的产物具有化学尺度的均 匀分散性以及高纯度。但由于反应周期长, 炦烧过 程会产生团聚，因此通常与其它合成方法例如模板 法等相结合使用 ${ }^{[22]}$, 以改善溶胶一凝胶法自身的不 足, 制备出较为完善、规整的纳米材料。Wang 等 ${ }^{[23]}$ 将溶胶 - 凝胶法与模板法相结合，首先配制 $\mathrm{Mn}\left(\mathrm{CH}_{3} \mathrm{COO}\right)_{2}$ 、柠檬酸和正丙醇的混合溶液, $\mathrm{Mn}^{2+}$ 与柠檬酸的摩尔比为 $1: 2$, 通过添加 $\mathrm{NH}_{3} \cdot \mathrm{H}_{2} \mathrm{O}$ 调节

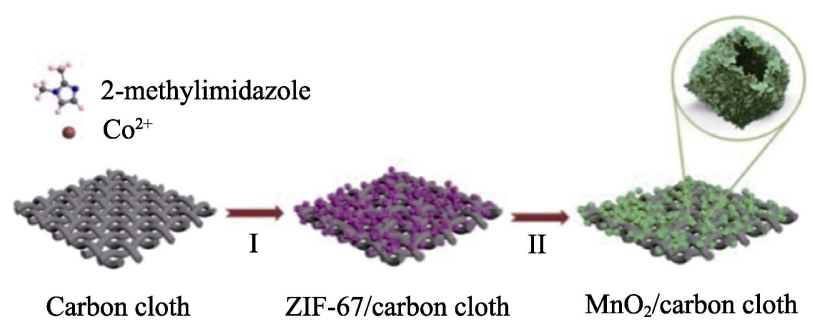

图 1 在碳布上两步合成 $\mathrm{MnO}_{2}$ 空心多面体纳米结构的制备 过程示意图 ${ }^{[13]}$

Fig. 1 Schematic illustration for the two-step preparation process of $\mathrm{MnO}_{2}$ hollow polyhedrons nanostructures assembled on carbon cloth $^{[13]}$
$\mathrm{pH}$ 至 8.5 。将该混合溶液在 $80{ }^{\circ} \mathrm{C}$ 下加热醚化，提取 过量的正丙醇。当溶液变成透明的粘性溶胶时，将 溶胶倒入制备好的多孔氧化铝(AAO)中, $80{ }^{\circ} \mathrm{C}$ 常压 干燥 $2 \mathrm{~h}, 400{ }^{\circ} \mathrm{C}$ 加热 $12 \mathrm{~h}$, 得到 $\mathrm{MnO}_{2}$ 纳米线。图 $2(\mathrm{a}, \mathrm{b})$ 分别是利用磷酸将 $\mathrm{AAO}$ 扩孔 $20 \mathrm{~min}$ 获得的 模板 $\mathrm{A}$ 和扩孔 $40 \mathrm{~min}$ 获得的模板 $\mathrm{B}$ 生成的 $\mathrm{MnO}_{2}$ 纳米线的扫描电镜照片。赵娜英等 ${ }^{[24]}$ 配制 $\mathrm{Mn}\left(\mathrm{CH}_{3} \mathrm{COO}\right)_{2}$ 、柠檬酸和 $\mathrm{La}\left(\mathrm{NO}_{3}\right)_{3} \cdot 6 \mathrm{H}_{2} \mathrm{O}$ 混合溶液, 并用 $\mathrm{NH}_{3} \cdot \mathrm{H}_{2} \mathrm{O}$ 调节溶液 $\mathrm{pH}$ 至 6 , 在 $80{ }^{\circ} \mathrm{C}$ 加热、 $110{ }^{\circ} \mathrm{C}$ 脱水, 煅烧 $10 \mathrm{~h}, 90{ }^{\circ} \mathrm{C}$ 酸化, 洗涤至中性后, 干燥，获得锞掺杂 $\mathrm{MnO}_{2}$ 复合材料。李哲等 ${ }^{[25]}$ 以 $\mathrm{Mn}\left(\mathrm{CH}_{3} \mathrm{COO}\right)_{2}$ 、柠檬酸和硅藻土作为原料, 用溶胶凝胶法制备出纳米 $\mathrm{MnO}_{2}$ 和纳米 $\mathrm{MnO}_{2}$ 负载硅藻土 的复合材料并将其用于吸附苯酚。

\section{3 化学沉淀法}

化学沉淀法在高纯度纳米粒子液相合成中应用 较为广泛，是指在水溶液中通过调节反应条件将溶 解于其中的金属盐转变为难溶化合物或水合氧化物 而析出, 经过进一步处理得到纳米材料的方法 ${ }^{[26]}$ 。 化学沉淀法包括共沉淀法、均匀沉淀法、氧化水解 法、还原法等。化学沉淀法有反应温度较低、操作 简单、成本低等优点, 但也存在诸如生成物均匀性 较差、易发生硬团聚等缺点。通过改变反应物浓度、 调节溶液 $\mathrm{pH}$ 可以有效改善这一情况, 化学沉淀法 为批量生产纳米材料提供了很好的选择。 $\mathrm{Li}$ 等 ${ }^{[27]}$ 利用化学氧化聚合法获得 $\mathrm{Ti}_{3} \mathrm{C}_{2} \mathrm{~T}_{x} @ \mathrm{PDA}$ (聚多巴胺) 粉末, 将其溶于超纯水中, 加入 $\mathrm{KMnO}_{4}$ 溶液, 以十 六烷基三甲基溴化铵 (CTAB)和聚乙二醇( $\mathrm{PEG}$ )作为 表面活性剂，利用液相共沉淀法制备不同形貌的 $\mathrm{Ti}_{3} \mathrm{C}_{2} \mathrm{~T}_{x} @ \mathrm{MnO}_{2}$ 复合材料。其中 $\mathrm{MnO}_{2}$ 的形貌包括纳 米片状、米粒状、纳米花状、纳米线状。米粒状 $\mathrm{MnO}_{2}$ 长度约为 $100 \mathrm{~nm}$, 纳米片状则可以在表面活 性剂的作用下团聚成纳米花状，其直径为 $100 \mathrm{~nm}$ 。 Mahamallik 等 ${ }^{[28]}$ 利用 $\mathrm{KMnO}_{4}$ 和 $\mathrm{MnCl}_{2} \cdot 4 \mathrm{H}_{2} \mathrm{O}$ (摩尔
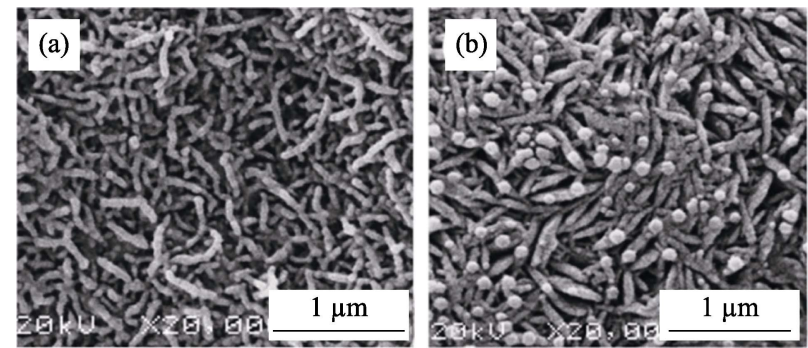

图 2 采用溶胶一凝胶法与模板法相结合, 以(a) AAO 模板 A 和(b) AAO 模板 B 制备的纳米 $\mathrm{MnO}_{2}$ 的扫描电镜照片 ${ }^{[23]}$

Fig. 2 SEM images of nanostructured $\mathrm{MnO}_{2}$ fabricated by using Sol-Gel and template methods with (a) AAO template A and (b) AAO template $\mathrm{B}^{[23]}$ 
比 2:3)通过氧化还原共沉淀法合成了 $\mathrm{MnO}_{2}$ 纳米 片。在磁力搅拌下, 将 $\mathrm{KMnO}_{4}$ 溶液 $(2 \mathrm{mmol} / \mathrm{L}, 100 \mathrm{~mL}$ ) 以 $5 \mathrm{~mL} / \mathrm{min}$ 的速率滴加入 $\mathrm{MnCl}_{2}$ 溶液 $(3 \mathrm{mmol} / \mathrm{L}$, $100 \mathrm{~mL}$ )中, 分离出棕色沉淀, 在 $90{ }^{\circ} \mathrm{C}$ 温度下干燥 $4 \mathrm{~h}$, 再一次将干燥的固体通过摚拌重新分散在水中, 经适当清洗得到上清液, 用离心法将物料从液体中 分离出来, 在 $90{ }^{\circ} \mathrm{C}$ 下干燥 $12 \mathrm{~h}$, 即得到具有微孔结 构的 $\mathrm{MnO}_{2}$ 纳米片, 其比表面积为 $301 \mathrm{~m}^{2} / \mathrm{g}$, 孔径为 $0.364 \mathrm{~nm}$ (图 3)。

\section{4 固相合成法}

固相合成法是采用较低温度通过固化反应生成 纳米二氧化锰的合成方法, 反应过程主要是通过固 体反应物高速球磨等。因实验方法简单、环境污染 较小、生成物产量高、反应选择性较好, 固相合成 法为纳米材料的合成提供了一种简单、高效、低价 的方法。但由于二氧化锰本身的固有特性, 采用此 方法不宜合成单晶型的纳米级产物。吴吴天等 ${ }^{[29]}$ 按 照一定比例混合 $\mathrm{KMnO}_{4}$ 和 $\mathrm{MnCl}_{2} \cdot 4 \mathrm{H}_{2} \mathrm{O}$, 在研钵中 研细, $80{ }^{\circ} \mathrm{C}$ 水浴 $3 \mathrm{~h}$, 离心洗涤后, $80{ }^{\circ} \mathrm{C}$ 烘干获得 纳米棒状 $\mathrm{MnO}_{2}$ 。随着反应物 $\mathrm{MnCl}_{2} \cdot 4 \mathrm{H}_{2} \mathrm{O}$ 比例的不 断增大, 获得纳米棒状 $\mathrm{MnO}_{2}$ 的直径与长度都不断 增大, $\mathrm{KMnO}_{4}$ 和 $\mathrm{MnCl}_{2} \cdot 4 \mathrm{H}_{2} \mathrm{O}$ 的摩尔比为 $2: 2 、 2: 3$ 、 $2: 4$ 时产物的长径比分别为 7、9、12。龚良玉 ${ }^{[30]}$ 利 用表面活性剂辅助固相合成法制备了直径为 10 $15 \mathrm{~nm}$ 、长度为 $100 \sim 150 \mathrm{~nm}$ 的 $\alpha-\mathrm{MnO}_{2}$ 纳米棒。李 娟等 ${ }^{[31]}$ 将摩尔比相同的 $\mathrm{Mn}\left(\mathrm{CH}_{3} \mathrm{COO}\right)_{2}$ 与柠檬酸混 合均匀, 于玛瑙研针中研磨 $30 \mathrm{~min}$ 以上。待体系颜
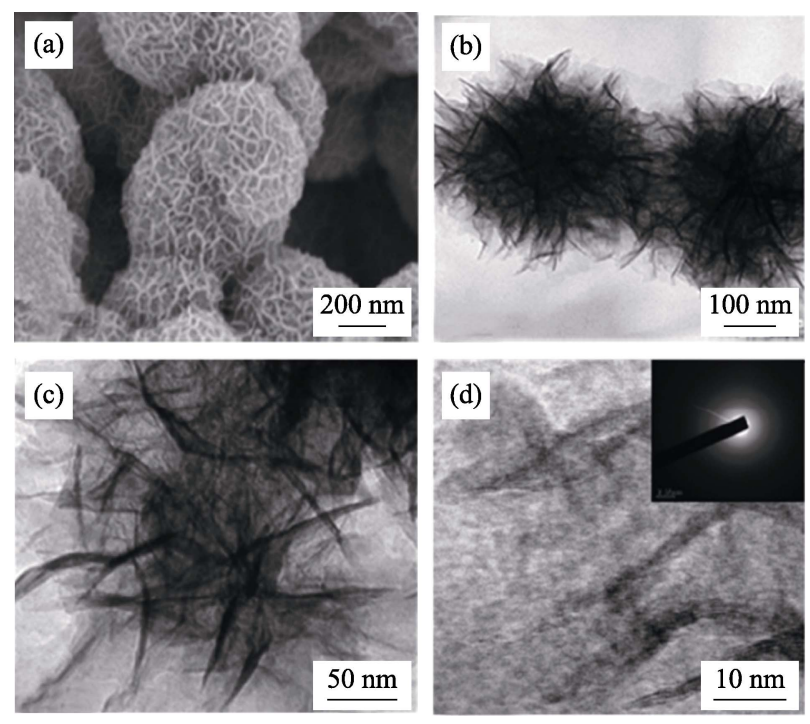

图 $3 \mathrm{MnO}_{2}$ 纳米片组装体的(a) FESEM、(b, c) TEM 和 (d) HRTEM 照片, (d)中插图为 SAED 花样 ${ }^{[28]}$

Fig. 3 (a) FESEM, (b, c) TEM and (d) HRTEM images of $\mathrm{MnO}_{2}$ assembled nanosheets with inset in (d) showing the corresponding SAED pattern ${ }^{[28]}$
色不再变化时, 在 $50 \sim 60{ }^{\circ} \mathrm{C}$ 反应 $10 \mathrm{~h}$, 在马弗炉中 $300{ }^{\circ} \mathrm{C}$ 煅烧 $10 \mathrm{~h}$ 。之后在 $2 \mathrm{~mol} / \mathrm{L} \mathrm{H}_{2} \mathrm{SO}_{4}$ 中于 $80 \sim 90{ }^{\circ} \mathrm{C}$ 酸化处理 1 2 h, 冷却后用蒸馏水洗涤至中 性, $105{ }^{\circ} \mathrm{C}$ 烘干 $10 \mathrm{~h}$, 取出研细, 所得产物是粒径为 20 30 nm 的球形纳米 $\gamma-\mathrm{MnO}_{2}$ 颗粒。

\section{2 纳米二氧化锰的应用}

\section{1 储能电极}

二氧化锰可作为电池的正极材料 ${ }^{[32]}$, 较广泛地 应用于锌/二氧化锰电池、镁/二氧化锰电池和碱性 锌/二氧化锰电池等。因二氧化锰的电容性较好, 在 超级电容器中可替代高价的氧化钉作为电极材料。 Wang 等 ${ }^{[16]}$ 利用微波辅助水热法制备直径为 $200 \mathrm{~nm}$ 的纳米花状 $\delta-\mathrm{MnO}_{2}$, 并将其作为超级电容器的电 极材料。在 $1 \mathrm{~mol} / \mathrm{L} \mathrm{Na}_{2} \mathrm{SO}_{4}$ 电解质溶液中, $2.1 \mathrm{~A} / \mathrm{g}$ 的电流密度下测得其电容与循环性能如图 4 所示。 $\delta-\mathrm{MnO}_{2}$ 在初始 400 次循环中比电容快速降低, 可能 是由于材料的循环收缩和膨胀以及材料在集流器上 的弱结合造成电极中松散材料出现损失。经过 400 次循环后, 材料的比电容保持相对稳定。经过 5000 次循环后, 比电容为初始值的 $89 \%$, 表明该材料具 有良好的充放电循环稳定性。Shafi 等 ${ }^{[33]}$ 用化学沉淀 法合成了 $\alpha-\mathrm{MnO}_{2}$ 纳米晶, 对制备的样品进行不同 温度的退火处理, 并进行电化学性能测试。结果显 示, $\mathrm{MnC}_{4.5}$ 即纯相 $\alpha-\mathrm{MnO}_{2}$ 纳米晶是超级电容器较佳 的电极材料。在 $\mathrm{MnO}_{2}$ 的不同晶型中, $\alpha-\mathrm{MnO}_{2}$ 在超 级电容器中应用较为广泛。近年来随着技术的不断 进步, 纳米 $\mathrm{MnO}_{2}$ 在电极材料方面的应用更加多样, 涵盖面也更加广泛 ${ }^{[34-36]}$ 。由于二氧化锰的晶型复杂, 随着反应条件的改变极易发生变化, 且随着对储能

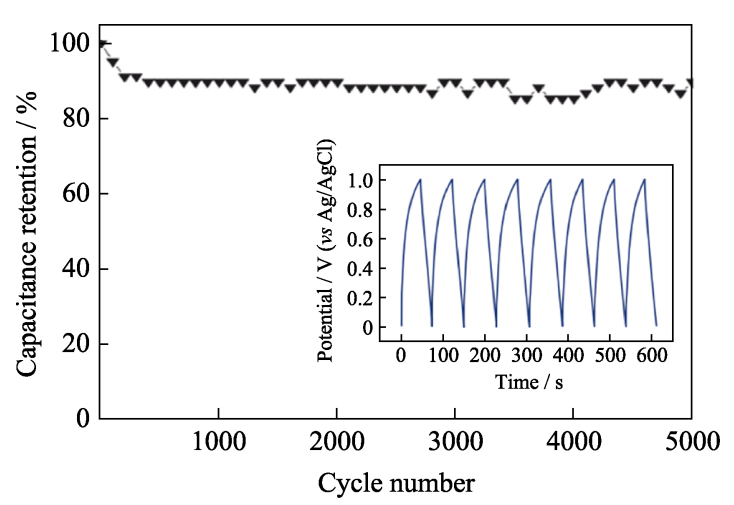

图 4 在 $1 \mathrm{~mol} / \mathrm{L} \mathrm{Na}_{2} \mathrm{SO}_{4}$ 电解质溶液、2.1 A/g 电流密度下 $\delta-\mathrm{MnO}_{2}$ 的电容保持率, 插图为相应的 $\delta-\mathrm{MnO}_{2}$ 的充放电曲线 ${ }^{[16]}$ Fig. 4 Capacitance retention of $\delta-\mathrm{MnO}_{2}$ at current density of $2.1 \mathrm{~A} / \mathrm{g}$ in $1 \mathrm{~mol} / \mathrm{L} \mathrm{Na}_{2} \mathrm{SO}_{4}$ electrolyte with inset showing the corresponding charge-discharge curves ${ }^{[16]}$ 
材料性能要求的不断提高, 单一材料已不能满足当 前对能量密度等的需求, 调控二氧化锰的晶型和制 备复合材料成为当前的研究重点。含锰复合氧化物 作为电池正极材料的应用较广泛, 其中 $\mathrm{LiMn}_{2} \mathrm{O}_{4}$ 正 极材料为尖晶石结构, 理论容量为 $148 \mathrm{mAh} / \mathrm{g}$; 以 高纯度的 $\mathrm{Mn}_{3} \mathrm{O}_{4}$ 作为原料, 可进一步提高其容量并 改善循环稳定性, 主要是因为 $\mathrm{Mn}_{3} \mathrm{O}_{4}$ 与 $\mathrm{LiMn}_{2} \mathrm{O}_{4}$ 有 类似的结构, 制备过程中结构不会发生剧烈变化 ${ }^{[37]}$ 。 $\mathrm{LiMnPO}_{4}$ 正极材料为橄榄石结构, 结构较稳定, 能 量密度与放电平台均比相同结构的 $\mathrm{LiFePO}_{4}$ 高, 但 其导电性能与倍率性能较差, 循环稳定性不高 ${ }^{[38]}$ 。针 对上述问题，科研工作者发现通过纳米化以及对晶 面进行篮选可以改善这一情况, 从而提高其电化学 性能 ${ }^{[39]}$ 。上述材料作为锂离子动力电池的正极材料 已有产业化应用，在新能源汽车领域具有良好的应 用前景。

\section{2 电致变色器件}

电致变色是一种通过外加电压实现离子的注入 与抽出, 从而达到颜色变化的现象 ${ }^{[40-41]}$ 。由于电致 变色材料具有在电场作用下改变颜色的特性, 在智
能窗 ${ }^{[2-44]}$ 、防眩后视镜 ${ }^{[45]}$ 、显示器 ${ }^{[46]}$ 以及防眩滑雪 镜等方面均具有广泛的应用。纳米二氧化锰自身价 态丰富, 颗粒尺寸较小, 比表面积较大, 离子、电子 传输速率快，因而适合应用于电致变色器件。但由 于纯二氧化锰本身的颜色主要是在棕色和黄色之间 变化, 光调制幅度较小, 需要将其与其它光调制幅 度较大的材料进行复合。Zhou 等 ${ }^{[47]}$ 利用恒电位阳极 电沉积法制备聚苯胺/二氧化锰 $\left(\mathrm{PANI} / \mathrm{MnO}_{2}\right)$ 杂化电 致变色薄膜。PANI 的电聚合和 $\mathrm{MnO}_{2}$ 的电沉积同时 发生, 合成的纳米 $\mathrm{MnO}_{2}$ 可以作为氧化剂, 进一步 改善 PANI 的化学聚合, 促进杂化膜的快速生长。这 种独特的沉积机制产生的 $\mathrm{PANI} / \mathrm{MnO}_{2}$ 杂化电致变 色薄膜较纯 PANI 膜在形貌、结构、电化学和电致 变色性能上有巨大差异, 显示出更高的光学对比 度、着色效率和循环稳定性(图 5)。杂化膜的优异性 能可以归功于其相互连接的纳米粒子的多孔形貌, 以及 PANI 与 $\mathrm{MnO}_{2}$ 之间的施主一受体相互作用。 Sakai 等 ${ }^{[48]}$ 通过将层状质子锰氧化物 $\mathrm{H}_{0.13} \mathrm{MnO}_{2} \cdot 0.7 \mathrm{H}_{2} \mathrm{O}$ 降解为胶体单层，合成了厚度为 $0.5 \mathrm{~nm}$ 左右的 $\mathrm{MnO}_{2}$ 纳米片。通过静电自组装的方法, 将 $\mathrm{MnO}_{2}$ 纳
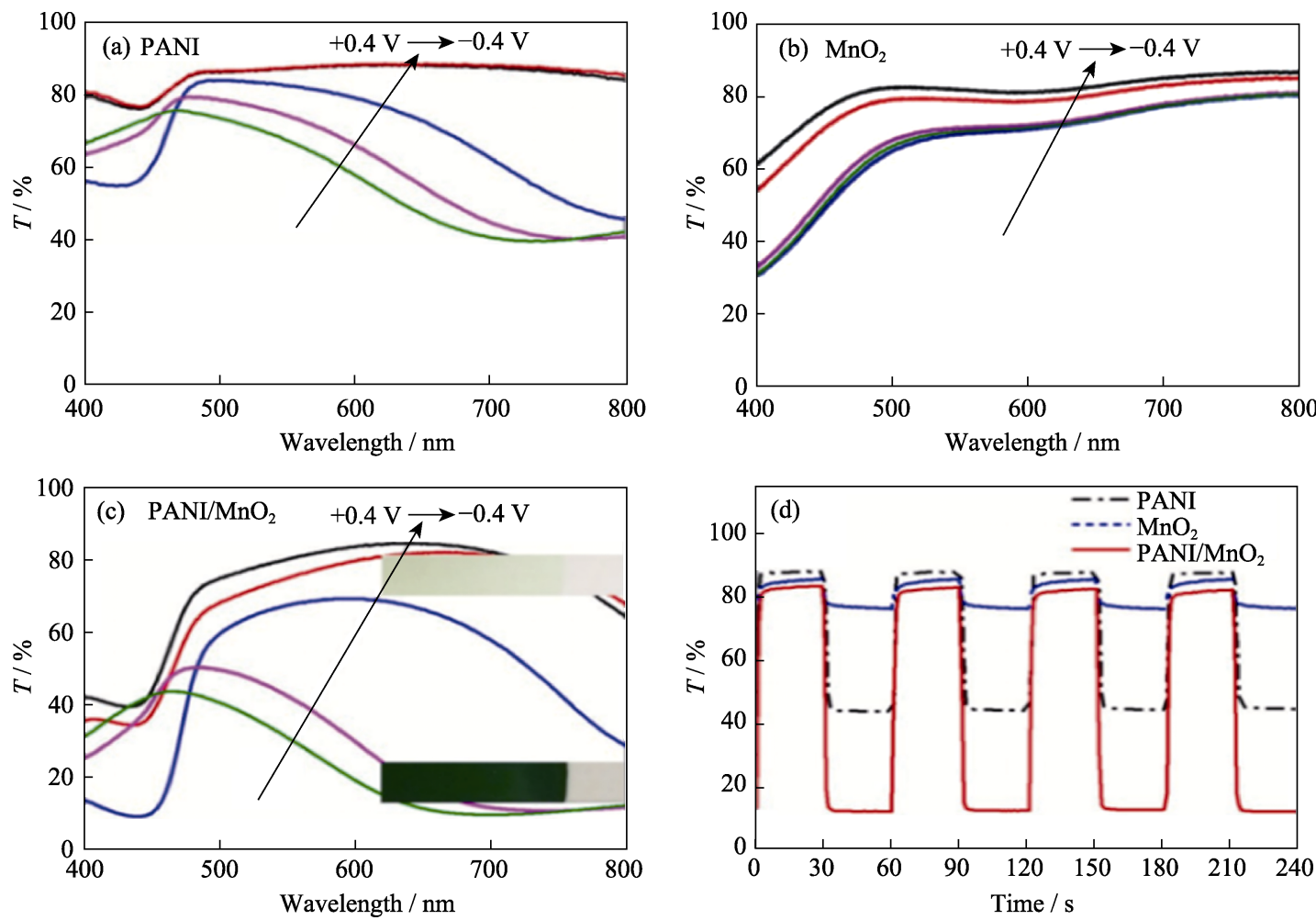

图 5 (a) PANI、 (b) $\mathrm{MnO}_{2}$ 和(c) $\mathrm{PANI} / \mathrm{MnO}_{2}$ 在不同电压下的紫外-可见光透过率光谱, (c)中的插图为 $\mathrm{PANI} / \mathrm{MnO}_{2}$ 杂化膜电沉积在 ITO/玻璃上的褪色态(上, 浅绿黄色)和着色态(下, 深青绿色)的照片; (d) $\mathrm{PANI} \mathrm{MnO}_{2}$ 和 $\mathrm{PANI} / \mathrm{MnO}_{2}$ 杂化膜在 $\lambda_{680 \mathrm{~nm}}(-0.4 \mathrm{~V} /+0.4 \mathrm{~V}$, 每周期 $60 \mathrm{~s})$ 的响应时间曲线 ${ }^{[47]}$

Fig. 5 UV-Vis transmittance spectra of (a) PANI, (b) $\mathrm{MnO}_{2}$, and (c) $\mathrm{PANI} / \mathrm{MnO}_{2}$ at different potentials with insets in (c) showing the photos of PANI/ $\mathrm{MnO}_{2}$ hybrid film electrodeposited on ITO/glass at bleached (upper, light greenish yellow) and colored state (lower, dark bluish green), and (d) switching curves comparison between PANI, $\mathrm{MnO}_{2}$, and PANI $/ \mathrm{MnO}_{2}$ hybrid films at $\lambda_{680 \mathrm{~nm}}(-0.4 \mathrm{~V} /+0.4 \mathrm{~V}, 60 \mathrm{~s} / \text { cycle })^{[47]}$ 
米片沉积在氧化铟锡(ITO)透明导电玻璃上。相关电 化学测试表明, 随着 $\mathrm{MnO}_{2}$ 纳米片层数的增加, 氧 化还原峰线性增强。尽管纳米片的规则堆积结构在 最初的几个扫描周期中已经崩溃, 但是纳米片本身 对 $\mathrm{CV}$ 循环保持稳定。 $\mathrm{MnO}_{2}$ 纳米片电极表现出阳极 电致变色, 着色效率高。吸光度的变化与 $\mathrm{MnO}_{2}$ 纳 米片的层数成正比, 通过控制 $\mathrm{MnO}_{2}$ 纳米片的层数, 可以很容易地实现光密度的调控。Falahatgar 等 ${ }^{[49]}$ 采用浸涂溶胶一凝胶法在 $300{ }^{\circ} \mathrm{C}$ 的空气中, 于 ITO 玻璃上沉积了 $\mathrm{Zn}$ 和 $\mathrm{Mn}$ 摩尔比分别为 $8 \%$ 、16\%和 $25 \%$ 的 $\mathrm{MnO}_{2}-\mathrm{ZnO}$ 纳米颗粒薄膜样品, 对薄膜的结 构、表面、光学和电致变色性能进行了研究。测试 结果表明, 制备的样品为无定形态, 在 20 和 $50 \mathrm{mV} / \mathrm{s}$ 的扫描速率下, 薄膜的电致变色性能取决于 $\mathrm{Zn}$ 与 $M n$ 的组成比。

\section{3 催化剂}

由于纳米二氧化锰具有良好的氧化还原特性以 及特有的纳米尺寸效应, 因此在催化领域有较为广 泛的应用。不同形貌的纳米二氧化锰的吸附效果有 所差异并且对吸附物的影响较大, 目前主要是对反 应条件进行精细化的控制, 生成特定的形貌从而满 足相关催化需求。Lyu 等 ${ }^{[50]}$ 采用共沉淀法合成了 $\mathrm{MgAl}$ 层状双氢氧化物(LDHs)。LDHs 在 $550{ }^{\circ} \mathrm{C}$ 的 空气中㷽烧 $10 \mathrm{~h}$ 获得层状双氧化物(LDOs), 将制备 的 LDOs 加入䘪丙基三甲氧基硅烷(MPTS)甲苯溶液 中, 在 $\mathrm{Ar}$ 气氛中回流, 过滤得到固体。用乙醇洗涤 固体, 在 $80{ }^{\circ} \mathrm{C}$ 下干燥, 得到 $\mathrm{LDO}-\mathrm{Si}(\mathrm{SH})$ 。将 LDO-Si(SH)或 LDO 分散于 $0.1 \mathrm{~mol} / \mathrm{L} \mathrm{KMnO}_{4}$ 水溶液 中, 然后在 $\mathrm{Ar}$ 气氛、 $80{ }^{\circ} \mathrm{C}$ 下剧烈搅拌。过滤后的 固体用热去离子水洗涤, 然后在 $90{ }^{\circ} \mathrm{C}$ 下真空干燥 $4 \mathrm{~h}$, 得到 LDH-Si(SH)-Mn 或 LDH-Mn。LDH-Mn 和 LDH-Si(SH)-Mn 具有显著的乙苯转化率, 表明 $\mathrm{Mn}$ 物种主要负责氧化活性。在没有过氧化氢叔丁基 (TBHP)作为引发剂的情况下, LDH-Si(SH)-Mn 的乙 苯转化率仅为 $0.7 \%$, 而添加少量 TBHP 后转化率达 到 $29.5 \%$, 说明 TBHP 起到了启动催化剂的作用。

Wang 等 ${ }^{[1]}$ 利用水热法和 $\mathrm{CCl}_{4}$ 溶剂法分别制备了纳 米棒状、类丝状、管状 $\alpha-\mathrm{MnO}_{2}$ 和花状球形 $\mathrm{Mn}_{2} \mathrm{O}_{3}$, 如图 6(a)所示。各个样品的催化性能为棒状 $\alpha-\mathrm{MnO}_{2}>$ 管状 $\alpha-\mathrm{MnO}_{2}>$ 花状 $\mathrm{Mn}_{2} \mathrm{O}_{3}>$ 线状 $\alpha-\mathrm{MnO}_{2}$, 如图 6(b) 所示。 $\alpha-\mathrm{MnO}_{2}$ 纳米棒的优异的催化性能可能与高氧 含量和良好的低温还原性有关, 这种一维锰氧化物材 料在催化消除空气污染物中有较广阔的发展前景。

\section{4 生物传感器}

生物传感器是一种结合对生物或生物衍生敏感 元件的精密分析器件, 与理化换能器产生间断或连
续的数字信号, 被分析物的含量由信号强度与被分 析物成比例关系来确定。纳米二氧化锰由于具有宏 观量子隧道效应、小尺寸效应等纳米效应, 能够增 大电流响应, 降低检出限, 使检测的灵敏度大大提 高, 近年来在生物传感器领域逐渐被大家重视并得 到广泛应用。为了使生物传感器中的二氧化锰更好 地与检测物接触, 可以将它与比表面积较大的其它 材料进行复合或提高其自身的比表面积。Qi 等 ${ }^{[52]}$ 以 $\mathrm{MnO}_{2}$ 纳米片作为辅助, 利用配体-DNA 相互作用 和基于苂光偏振的方法来检测 $\mathrm{Ag}^{+}$。在预先形成的 普罗黄素(配体)-DNA 复合物中加入 $\mathrm{Ag}^{+}$可诱导释放 普罗黄素, 从而引起菼光极化的微弱变化, $\mathrm{MnO}_{2}$ 纳 米片可以放大这种荧光变化。图 7 表明, 荧光偏振
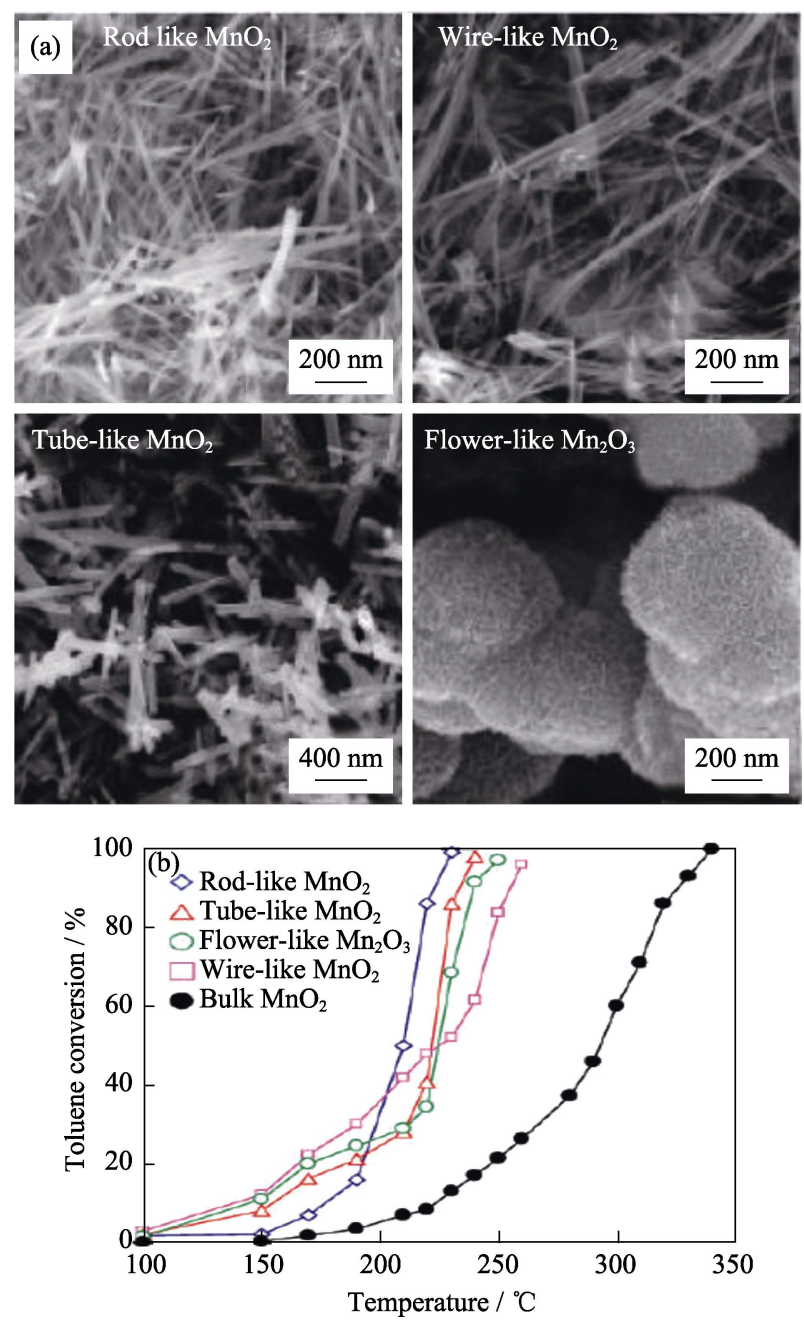

图 6 (a)纳米棒状、类丝状、管状 $\alpha-\mathrm{MnO}_{2}$ 和花状球形 $\mathrm{Mn}_{2} \mathrm{O}_{3}$ 的 SEM 照片; (b)在甲苯浓度为 $10^{-3}$ 、甲苯 $/ \mathrm{O}_{2}$ 的摩尔比为 $1 / 400$ 和空速为 $20000 \mathrm{~mL} /(\mathrm{g} \cdot \mathrm{h})$ 的条件下甲苯的转化率随反 应温度的变化曲线 ${ }^{[51]}$

Fig. 6 (a) SEM images of rod-like $\alpha-\mathrm{MnO}_{2}$, wire-like $\alpha-\mathrm{MnO}_{2}$, tube-like $\alpha-\mathrm{MnO}_{2}$, and flower-like $\mathrm{Mn}_{2} \mathrm{O}_{3}$; (b) Toluene conversion as a function of reaction temperature over the catalysts under the conditions of a toluene concentration of $10^{-3}$, toluene/ $\mathrm{O}_{2}=1 / 400(\mathrm{~mol} / \mathrm{mol})$, and a space velocity of $20000 \mathrm{~mL} /(\mathrm{g} \cdot \mathrm{h})^{[51]}$ 


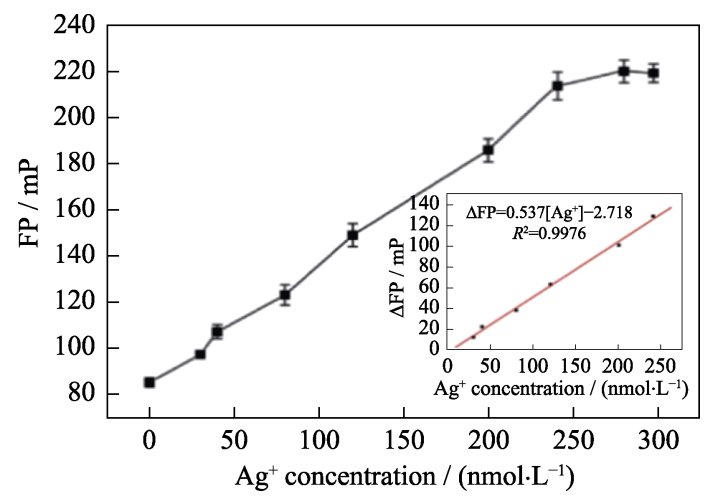

图 7 在 $\mathrm{MnO}_{2}$ 纳米片 $(80 \mu \mathrm{g} / \mathrm{mL})$ 存在下, 加入不同浓度 $\mathrm{Ag}^{+}$ 的荧光偏振值(FP), 插图为 $\Delta \mathrm{FP}$ 与 $\mathrm{Ag}^{+}$的浓度之间的线性关 系 ${ }^{[52]}$

Fig. 7 Measurement of FP following the addition of various concentrations of $\mathrm{Ag}^{+}$in the presence of $\mathrm{MnO}_{2}$ nanosheets $(80 \mu \mathrm{g} / \mathrm{mL})$ with inset showing the linear relationship between $\Delta \mathrm{FP}$ and $\mathrm{Ag}^{+}$concentration ${ }^{[52]}$

差值 $(\Delta \mathrm{FP})$ 与 $\mathrm{Ag}^{+}$的浓度之间具有良好的线性关系。 该方法进一步阐明存在 $\mathrm{MnO}_{2}$ 纳米片时加入 $\mathrm{Ag}^{+}$的 过程中引起苂光偏振变化的机理。这些研究表明, 目前的 $\mathrm{MnO}_{2}$ 纳米片辅助荧光偏振生物传感器是一 种很有前途的检测环境水样中 $\mathrm{Ag}^{+}$的工具。Xiao 等 ${ }^{[53]}$ 制备了一种基于 $\mathrm{PtAu}$ 合金和 $\mathrm{MnO}_{2}$ 二元纳米复合材 料修饰的石墨烯纸的新型柔性电化学传感器。通过 一步无模板电沉积在祄底上生长珊瑚状 PtAu- $\mathrm{MnO}_{2}$ 纳米复合材料, 使 $\mathrm{PtAu}$ 合金、 $\mathrm{MnO}_{2}$ 与基体密切接 触。柔性电极具有独特的结构和电化学性能, 如更 好的均匀性、更大的活性比表面积和更快的电子转 移。在非酶葡萄糖检测中, PtAu- $\mathrm{MnO}_{2}$ 二元纳米结构 修饰的石墨烯纸表现出良好的传感性能。

\section{3 结语}

虽然纳米二氧化锰的制备及应用取得了显著进 展, 但仍需要研究纳米二氧化锰的可控制备方法及 制备与性能之间的关系, 以期进一步提升性能, 满 足更高的使用要求。当前含锰氧化物在电池正极材 料的应用已经进入了产业化阶段。现有新能源汽车 的发展大幅度提升了对电池正极材料的能量密度的 要求, 而锂电池在向着高容量、低成本的发展过程 中含锰复合材料起着重要推动作用。由于二氧化锰 的晶型多变、反应过程中存在副反应, 使其发展存 在诸多壁垒。为了改善二氧化锰材料的性能, 未来 除了体相掺杂、表面包覆、使用梯度材料等方法, 还 需要对材料的形貌与尺寸进行精细控制, 提高材料 的循环性能。未来纳米二氧化锰的研究与高容量富 锂正极/碳负极锂离子电池正极材料的发展密切相
关，将向着与其他材料复合的方向发展，从而更好 地满足正极材料对性能的需求。由于单一纳米二氧 化锰作为电致变色材料的光调制幅度较小, 未来主 要是与其它材料复合来增强材料的性能, 或者与光 调制幅度较大的材料制备互补型电致变色器件来改 善电致变色器件的性能。只有不断探索材料性能与 材料自身的尺寸、晶型、形貌等之间的关系，掌握 其规律，纳米二氧化锰才能被更好地开发与利用， 实现产业化应用。

\section{参考文献:}

[1] SUN K, LI S Y, WAIGI M G, et al. Nano- $\mathrm{MnO}_{2}$-mediated transformation of triclosan with humic molecules present: kinetics, products, and pathways. Environmental Science \& Pollution Research, 2018, 25(15): 14416-14425.

[2] SEO J K, SHIN J W, CHUNG H, et al. Intercalation and conversion reactions of nanosized $\beta-\mathrm{MnO}_{2}$ cathode in the secondary $\mathrm{Zn} / \mathrm{MnO}_{2}$ alkaline battery. The Journal of Physical Chemistry $C$, 2018, 122(21): 11177-11185.

[3] XUE F, WU S, WANG M X, et al. A three-dimensional graphene/ $\mathrm{CNT} / \mathrm{MnO}_{2}$ hybrid as supercapacitor electrode. Integrated Ferroelectrics, 2018, 190(1): 156-163.

[4] GU X, YUE J, LI L J, et al. General synthesis of $\mathrm{MnO}_{x}\left(\mathrm{MnO}_{2}\right.$, $\left.\mathrm{Mn}_{2} \mathrm{O}_{3}, \mathrm{Mn}_{3} \mathrm{O}_{4}, \mathrm{MnO}\right)$ hierarchical microspheres as lithium-ion battery anodes. Electrochimica Acta, 2015, 184: 250-256.

[5] FENG Q, KANOH H, OOI K. Manganese oxide porous crystals. Journal of Materials Chemistry, 1999, 9(2): 319-333.

[6] POST J E. Manganese oxide minerals: crystal structures and economic and environmental significance. Proceedings of the National Academy of Sciences, 1999, 96(7): 3447-3454.

[7] JIA Z J, WANG J, WANG Y, et al. Interfacial synthesis of $\delta-\mathrm{MnO}_{2}$ nano-sheets with a large surface area and their application in electrochemical capacitors. Journal of Materials Science \& Technology, 2016, 32(2): 147-152.

[8] HUANG Y J, LI W S. Preparation of manganese dioxide for oxygen reduction in zinc air battery by hydro thermal method. Journal of Inorganic Materials, 2013, 28(3): 341-346.

[9] WEN J G, RUAN X Y, ZHOU Z T. Characterization of $\mathrm{MnO}_{2}$ aerogels prepared via supercritical drying technique. Journal of Inorganic Materials, 2009, 24(3): 521-524.

[10] XIAO X Z, YI Q F. Synthesis and electochemical capacity of $\mathrm{MnO}_{2} / \mathrm{SMWCNT/PANI}$ ternarycomposites. Journal of Inorganic Materials, 2013, 28(8): 825-830.

[11] DARR J A, ZHANG J Y, MAKWANA N M, et al. Continuous hydrothermal synthesis of inorganic nanoparticles: applications and future directions. Chemical Reviews, 2017, 117(17): 11125-11238.

[12] ZHAO P, YAO M Q, REN H B, et al. Nanocomposites of hierarchical ultrathin $\mathrm{MnO}_{2}$ nanosheets/hollow carbon nanofibers for high-performance asymmetric supercapacitors. Applied Surface Science, 2019, 463: 931-938.

[13] WU F F, GAO X B, XU X L, et al. Boosted Zn storage performance of $\mathrm{MnO}_{2}$ nanosheet-assembled hollow polyhedron grown on carbon cloth via a facile wet-chemical synthesis. ChemSusChem, 2020, 13(6): 1537-1545.

[14] LU C J, ZHU F Q, YIN J G, et al. Synthesis of $\alpha-\mathrm{MnO}_{2}$ nanowires via facile hydrothermal method and their application in $\mathrm{Li}-\mathrm{O}_{2}$ battery. Journal of Inorganic Materials, 2018, 33(9): 1029-1034.

[15] ZHU K, WANG C, CAMARGO P H C, et al. Investigating the effect of $\mathrm{MnO}_{2}$ band gap in hybrid $\mathrm{MnO}_{2}-\mathrm{Au}$ materials over the SPR-mediated activities under visible light. Journal of Materials 
Chemistry A, 2019, 7(3): 925-931.

[16] WANG L, MA W L, LI Y H, et al. Synthesis of $\delta-\mathrm{MnO}_{2}$ with nanoflower-like architecture by a microwave-assisted hydrothermal method. Journal of Sol-Gel Science and Technology, 2017, 82: 85-91.

[17] MA Z C, WEI X Y, XING S T, et al. Hydrothermal synthesis and characterization of surface-modified $\delta-\mathrm{MnO}_{2}$ with high Fenton-like catalytic activity. Catalysis Communications, 2015, 67: 68-71.

[18] LIU D Y, DONG L M, SHAN L W, et al. Preparation of $\mathrm{Fe}-\mathrm{MnO}_{2} / \mathrm{RGO}$ electrode and electrochemical properties. Ferroelectrics, 2019, 546(1): 41-47.

[19] XIE Y M, WANG L J, GUO Q Y, et al. Preparation of $\mathrm{MnO}_{2}$ /porous carbon material with core-shell structure and its application in supercapacitor. Journal of Materials Science Materials in Electronics, 2018, 29(10): 1-8.

[20] MATHUR A, HALDER A. One step synthesis of bifunctional iron-doped manganese oxide nanorods for rechargeable zinc-air batteries. Catalysis Science \& Technology, 2019, 9(5): 1245-1254.

[21] JITTIARPORN P, BADILESCU S, Al SAWAFTA M N, et al. Electrochromic properties of Sol-Gel prepared hybrid transition metal oxides - a short review. Journal of Science: Advanced Materials and Devices, 2017, 2(3): 286-300.

[22] MOHAMED M A, SALLEH W N W, JAAFAR J, et al. Carbon as amorphous shell and interstitial dopant in mesoporous rutile $\mathrm{TiO}_{2}$ : bio-template assisted Sol-Gel synthesis and photocatalytic activity. Applied Surface Science, 2017, 393: 46-59.

[23] WANG X Y, WANG X Y, HUANG W G, et al. Sol-Gel template synthesis of highly ordered $\mathrm{MnO}_{2}$ nanowire arrays. Journal of Power Sources, 2005, 140(1): 211-215.

[24] 赵娜英, 市洁鹏, 杨雪健, 等. 溶胶-凝胶法制备掺镧改性纳米 $\mathrm{MnO}_{2}$. 化工新型材料, 2019, 47(5): 164-171.

[25] 李哲, 汤化伟, 王百年. 纳米 $\mathrm{MnO}_{2}$ 负载硅藻土对苯酚废水的吸 附性能研究. 合肥工业大学学报(自然科学版), 2016, 39(5): 695-700.

[26] THEISS F L, AYOKO G A, FROST R L. Synthesis of layered double hydroxides containing $\mathrm{Mg}^{2+}, \mathrm{Zn}^{2+}, \mathrm{Ca}^{2+}$ and $\mathrm{Al}^{3+}$ layer cations by co-precipitation methods-a review. Applied Surface Science, 2016, 383: 200-213.

[27] LI X L, ZHU J F, JIAO Y H, et al. Manganese dioxide morphology on electrochemical performance of $\mathrm{Ti}_{3} \mathrm{C}_{2} \mathrm{~T}_{x} @ \mathrm{MnO}_{2}$ composites. Journal of Inorganic Materials, 2020, 35(1): 119-125.

[28] MAHAMALLIK P, SAHA S, PAL A. Tetracycline degradation in aquatic environment by highly porous $\mathrm{MnO}_{2}$ nanosheet assembly. Chemical Engineering Journal, 2015, 276: 155-165.

[29] 吴吴天, 张振忠, 赵芳霞, 等. 低温固相法制备的纳米 $\alpha-\mathrm{MnO}_{2}$ 的性能. 电池, 2015, 45(3): 157-159.

[30] 枈良玉. 固相合成 $\mathrm{MnO}_{2}$ 纳米棒的电容性能及其 $\mathrm{PbO}$ 改性研究. 青岛农业大学学报(自然科学版), 2011, 28(2): 157-161.

[31] 李娟, 夏熙. 纳米 $\mathrm{MnO}_{2}$ 的固相合成及其电化学性能的研究(I): 纳 米 $\gamma-\mathrm{MnO}_{2}$ 的合成及表征. 高等学校化学学报, 1999, 20(9): 1434-1437.

[32] SHIN J, SEO J K, YAYLIAN R, et al. A review on mechanistic understanding of $\mathrm{MnO}_{2}$ in aqueous electrolyte for electrical energy storage systems. International Materials Reviews, 2019: 1-32.

[33] SHAFI P M, BOSE A C. Structural evolution of tetragonal $\mathrm{MnO}_{2}$ and its electrochemical behavior. AIP Conference Proceedings, 2016, 1731(1): 050038 .

[34] HAN S D, KIM S, LI D G, et al. Mechanism of Zn insertion into nanostructured $\delta-\mathrm{MnO}_{2}$ : a nonaqueous rechargeable $\mathrm{Zn}$ metal battery. Chemistry of Materials, 2017, 29(11): 4874-4884.

[35] ZERAATI A S, ARJMAND M, SUNDARARAJ U. Silver nanowire $/ \mathrm{MnO}_{2}$ nanowire hybrid polymer nanocomposites: materials with high dielectric permittivity and low dielectric loss. $A C S$
Applied Materials \& Interfaces, 2017, 9(16): 14328-14336.

[36] REHMAN S, TANG T Y, ALI Z, et al. Integrated design of $\mathrm{MnO}_{2} @$ carbon hollow nanoboxes to synergistically encapsulate polysulfides for empowering lithium sulfur batteries. Small, 2017, 13(20): 1700087.

[37] LUO P F, HUANG Z. Fabrication of scandium-doped lithium manganese oxide as a high-rate capability cathode material for lithium energy storage. Solid State Ionics, 2019, 338: 20-24.

[38] WANG Y M, WANG F, FENG X J. Porous nest-like LiMnPO 4 microstructures assembled by nanosheets for lithium ion battery cathodes. Journal of Materials Science: Materials in Electronics, 2018, 29(2): 1426-1434.

[39] 李俊豪, 冯斯桐, 张圣洁, 等. 高性能磷酸锰锂正极材料的研究 进展. 材料导报, 2019, 33(9): 2854-2861.

[40] ZHAO J X, WANG G H, ZHANG Q, et al. An underlying intercalation ion for fast-switching and stable electrochromism. Journal of Materials Science Materials in Electronics, 2019, 30(13): 12753-12756.

[41] LIU Y R, RYOTA S, CHEUK L H, et al. Electrochromic triphenylamine-based cobalt (II) complex nanosheets. Journal of Materials Chemistry C, 2019, 7(30): 9159-9166.

[42] CHEN C W, BRIGEMAN A N, HO T J, et al. Normally transparent smart window based on electrically induced instability in dielectrically negative cholesteric liquid crystal. Optical Materials Express, 2018, 8(3): 691.

[43] TONG Z Q, LIU S K, LI X G, et al. Achieving rapid Li-ion insertion kinetics in $\mathrm{TiO}_{2}$ mesoporous nanotube arrays for bifunctional high-rate energy storage smart windows. Nanoscale, 2018, 10: 3254-3261.

[44] CANNAVALE A, AYR U, FIORITO F, et al. Smart electrochromic windows to enhance building energy efficiency and visual comfort. Energies, 2020, 13(6): 1449.

[45] BECHINGER C, FERRERE S, ZABAN A, et al. Photoelectrochromic windows and displays. Nature, 1996, 383(6601): 608-610.

[46] CHO S I, KWON W J, CHOI S J, et al. Nanotube-based ultrafast electrochromic display. Advanced Materials, 2005, 17(2): 171-175.

[47] ZHOU D, CHE B Y, LU X H. Rapid one-pot electrodeposition of polyaniline/manganese dioxide hybrids: a facile approach to stable high-performance anodic electrochromic materials. Journal of Materials Chemistry C, 2017(5): 1758-1766.

[48] SAKAI N, EBINA Y, TAKADA K, et al. Electrochromic films composed of $\mathrm{MnO}_{2}$ nanosheets with controlled optical density and high coloration efficiency. Journal of the Electrochemical Society, 2005, 152(12): E384-E389.

[49] FALAHATGAR S S, GHODSI F E, TEPEHAN F Z, et al. Electrochromic performance of Sol-Gel derived amorphous $\mathrm{MnO}_{2}-\mathrm{ZnO}$ nanogranular composite thin films. Journal of Non-Crystalline Solids, 2015, 427: 1-9.

[50] LYU W M, YANG L, FAN B B, et al. Silylated MgAl LDHs intercalated with $\mathrm{MnO}_{2}$ nanowires: highly efficient catalysts for the solvent-free aerobic oxidation of ethylbenzene. Chemical Engineering Journal, 2015, 263: 309-316.

[51] WANG F, DAI H X, DENG J G, et al. Manganese oxides with rodwire-, tube-, and flower-like morphologies: highly effective catalysts for the removal of toluene. Environmental Science \& Technology, 2012, 46(7): 4034-4041.

[52] QI L, YAN Z, HUO Y, et al. $\mathrm{MnO}_{2}$ nanosheet-assisted ligand-DNA interaction-based fluorescence polarization biosensor for the detection of $\mathrm{Ag}^{+}$ions. Biosensors and Bioelectronics, 2017, 87: 566-571.

[53] XIAO F, LI Y Q, GAO H C, et al. Growth of coral-like PtAu- $\mathrm{MnO}_{2}$ binary nanocomposites on free-standing graphene paper for flexible nonenzymatic glucose sensors. Biosensors \& Bioelectronics, 2013, 41: 417-423. 